\title{
Malakoplakia of Gall Bladder: A Histological Surprise
}

\author{
Srinath Shankar Rathod ${ }^{1}$, Divya Dahiya ${ }^{2}$, Shibojit Talukder ${ }^{3 *}$ and Gopal Sharma ${ }^{4}$ \\ ${ }^{1}$ Senior Resident, Department Of General Surgery, PGIMER, Chandigarh, India \\ ${ }^{2}$ Associate Professor, Department of General Surgery, PGIMER, Chandigarh, India \\ ${ }^{3}$ Senior Resident, Department Of General Surgery, PGIMER, Chandigarh, India \\ ${ }^{4} J$ unior Resident, Department Of General Surgery, PGIMER, Chandigarh, India
}

Received: August 07, 2017; Accepted: September 05, 2017; Published: September 12, 2017

*Corresponding author: Shibojit Talukder, Senior Resident, Department Of General Surgery, PGIMER, Chandigarh, India, Tel: +919914017546; E-mail: shibojitsplace@gmail.com

\begin{abstract}
Malakoplakia of Gall bladder is a rare pathological entity with only a handful of cases reported in the literature. Clinically it can mimic carcinoma gall bladder or chronic cholecystitis. It can give rise diagnostic dilemma and influence intraoperative and postoperative decision making. It is almost never possible to make a pre-surgical diagnosis of gall bladder malakoplakia. Hence it remains as a histopathological surprise. Here, we describe two cases of Malakoplakia of gallbladder, both of which were presented as chronic cholecystitis and following pathological analysis of gall bladder specimen was proven to be malakoplakia.
\end{abstract}

Keywords: Malakoplakia; Gall Bladder Pathology

Abbreviations: USG: Ultra sonography; CECT: Contrast enhanced Computed Tomography

\section{Introduction}

Malakoplakia of gall bladder is reported rarely as a histological surprise in patients being operated for the diagnosis of either carcinoma gall bladder or chronic cholecystitis. It results from atypical chronic inflammatory response against pathogenic microorganism. Malakoplakia, xanthogranulomatous cholecystitis, aggressive inflammation and carcinoma gallbladder can produce similar features on imaging [1] thus making preoperative diagnosis is often impossible. To add to the diagnostic dilemma are the intraoperative features of hard mass with extensive infiltration into liver and surrounding organs such as duodenum and colon [2]. Surgeons would often consider carcinoma as the first differential and perform extensive resection to err on the safer side. Here we present two cases of Malakoplakia of gall bladder, both of which presented preoperatively as cholecystitis and one of them raised the suspicion of malignancy intraoperatively and prompted frozen section. In the same case, bile culture showed growth of Escherichia coli. Thus in such cases of equivocal diagnosis intraoperatively, we suggest use of frozen section and bile cultures that can guide postoperative therapy.

\section{Case history}

\section{Case 1:}

A 54-year-old hypertensive male was referred with complaint of intermittent pain in right upper quadrant for two years. Pain used to be aggravated by intake of fatty meal and also associated with nausea and vomiting. There was no history of anorexia or weight loss. Apart from being hypertensive, he had no other known comorbidity or any addiction. His general physical and abdominal examination was normal.

His preoperative blood work up was normal except a raised serum alkaline phosphatase level $(194 \mathrm{U} / \mathrm{ml})$. Ultrasonography (USG) abdomen showed slightly thickened gall bladder full of sludge with multiple calculi at neck. In view of USG findings, patient underwent contrast enhanced computed tomogram (CECT) abdomen which was suggestive of asymmetrical mural thickening of anterior wall of gall bladder. Thus he was planned for surgery with preoperative suspicion of carcinoma gall bladder.

Patient underwent diagnostic laparoscopy where in gall bladder was thickened and densely adhered to omentum. Gall bladder wall was seen infiltrating the liver. In view of above findings procedure was converted to open. Gall bladder wall was sent for frozen section which showed inflammatory cells comprising of plasma cells and lymphoid follicles with surrounding fibrosis and no evidence of malignancy. Intraop bile was also sent for culture that grown Escherichia coli. Postoperative period was uneventful and patient was discharged on culture based antibiotics on postoperative day 2. Follow up clinical examination and Ultrasonography was unremarkable at 6 months following surgery and patient is symptom free till date. On gross examination gall bladder was $7 \mathrm{~cm}$ long with congested and ulcerated mucosa. Cut surface showed a grayish white tumor-like nodule with areas of cholesterosis. On microscopic 
examination subepithelium showed diffuse sheets and clusters of foamy histiocytes admixed with many inflammatory cells comprising of lymphocytes, plasma cells, eosinophils and neutrophils. These foamy histiocytes showed many cytoplasmic eosinophilic globular bodies which were periodic acid schiff and Perl's positive suggestive of Michaelis-Gutman bodies. No Rokitansky-Aschoff Sinuses were seen (Figure 1).

\section{Case 2:}

The second case was of a 30-year-old lady who presented to us with complaint of recurrent attack of acute severe pain in right hypochondrium for 3 months. Her past medical, social and family history was unremarkable. Clinical examination was unremarkable.USG abdomen showed distended gall bladder with a single calculi in the region of gall bladder neck without any pericholecytic fluid. Patient was planned for Laparoscopic cholecystectomy. Patient underwent laparoscopic converted to open cholecystectomy due to altered Calot's triangle anatomy. Postoperative period was uneventful and patient was discharged on postoperative day 2. On gross examination of cut section of gall bladder was $6 \mathrm{~cm}$ in length with mucosal congestion at places. On Microscopy, gall bladder showed ulcerated mucosa with underlying granulation tissue infiltrating transmurally which is composed of predominantly foamy macrophages admixed with lymphocytes, plasma cells, eosinophils and focal areas of multinucleated giant cells. Foamy histiocytes contain oval to round structures that show strong positivity for periodic acid schiff, iron and von kossa stain features of Michaelis-Gutman bodies (Figure 1).
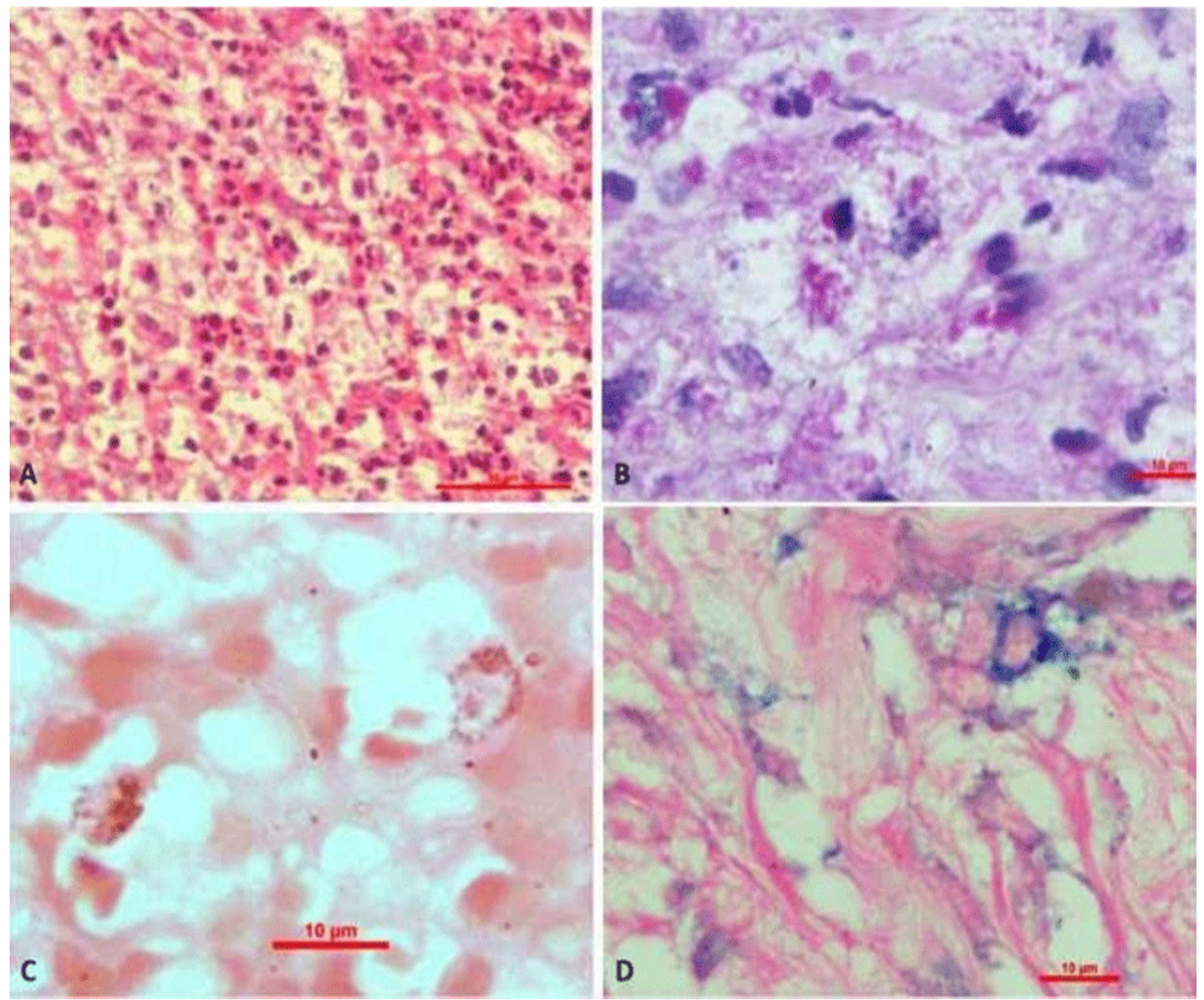

Figure 1: A panel of representative photomicrographs showing (A) High power picture of the mass showing sheets of histiocytes mixed with other inflammatory cells (Hematoxylin eosin, $\mathrm{x} 40$ ); (B) Same lesion on PAS staining to show the intracytoplasmic positively stained varying sized globules (x100); (C) Von Kossa staining to show the positively stained intracytoplasmic dark coloured fine calcium phosphate globules (x100) and (D) Perl's staining to show the varying sized intracytoplasmic blue coloured hemosiderin pigments (x100) 


\section{Discussion}

Malakoplakia was first described by Michaelis and Gutman [3] in 1902. It is a form of chronic granulomatous disease, usually affecting urinary tract [4]. It can also affect the other parts of the body such as gastrointestinal, genital, and biliary tracts, central nervous system and skin. Pathologically, on gross examination, Malakoplakia has characteristic appearance of soft yellow plaques or nodules [2]. Microscopically, it is characterized by the presence of sheets of mononuclear cells with granular cytoplasm (von Hansemann cells). Rokitansky-Aschoff sinuses seen in chronic cholecystitis, can sometimes mimic carcinoma. These sinuses have never been reported with malakoplakia and were not seen in our two cases as well. Michaelis-Gutman bodies considered pathognomic of this disorder are round to oval basophilic inclusion bodies found with inflammatory cells infiltrate.

Pathogenesis of this disorder is poorly understood with various mechanisms hypothesized such as the possible role of microbes [5] (such as Escherichia coli, Mycobacterium tuberculosis and Staphylococcus aureus), defective macrophage function due to abnormal micro tubular function and lysosomal activity resulting in incomplete destruction of bacteria. Calcium and iron deposition on these accumulating residual bacterial glycolipid wall results in the formation of basophilic round to oval intracytoplasmic and extracytoplasmic structures known as Michaelis-Gutmann bodies.

Xanthogranulomatous cholecystitis and malakoplakia of the gallbladder are considered to be a part of spectrum of similar pathology i.e. defective phagocytosis, that can present with different histological features [6]. Clinically it may be difficult to distinguish between more aggressive Malakoplakia and self-limited Xanthogranulomatous cholecystitis. Although Malakoplakia is considered a benign disease process for which local excision will suffice but it has been observed that many of these cases have aggressive disease process including high disease recurrence with fistulisation and partial or failure to respond to antibiotics.

Till now there have been 10 reported cases of Malakoplakia of Gall bladder in the literature. It was first described by Hanada et al [7]. They reported 17 cases of chronic cholecystitis with various granulomatous changes of the wall of gall bladder. The granuloma in one of their case contained typical Michaelis-Gutmann bodies. Tommaso et al [6] described another case of Malakoplakia of gall bladder arising in the setting of xanthogranulomatous cholecystitis and associated with infection. They documented bacilli around von hasemann cells on PAS staining. Our case represents the first case report of Malakoplakia of the gallbladder where in bile culture was positive for a postulated organism. Previous studies either showed bacilli like structures on staining [6] or were negative on staining or culture $[7,8]$.

Malakoplakia of gall bladder has always been an incidental histological finding. By mimicking carcinoma gall bladder Malakoplakia has influenced treatment protocol in several previous case reports $[1,2,9]$, like compelling frozen section as in our case or performing aggressive and extended resections. Post operatively Malakoplakia requires specific chemotherapy that concentrates in macrophages such as quinolone, trimethoprimsulfamethoxazole [2]. Bethanechol, a choline agonist, has been used in Malakoplakia [10] with the concept that bethanechol may correct the decreased cGMP levels that are important for microtubular and lysosomal function in macrophages [5]. Availability of culture report in first case, directed the choice of antibiotic, being both organism and macrophage specific.

\section{Declaration}

\section{Conflict Of Interest Statement:}

On behalf of all authors, the corresponding author states that there is no conflict of interest.

\section{Ethical Approval:}

This study involved human subject participation. All the procedures done to the patient namely surgery, investigations were carried out after obtaining valid informed consent from the patients as per institutional protocol. All the treatments provided were also in accordance with the institutional practice. All attempts have been made to prevent disclosure of identity and personal information of the patient. All procedures performed in studies involving human participants were in accordance with the ethical standards of the institutional and/or national research committee and with the 1964 Helsinki declaration and its later amendments or comparable ethical standards.

\section{References}

1. Hide G, Desai S, Bloxham CA. Malakoplakia of the gall-bladder: imaging and histological features. Clin Radiol. 2001;56(4):326-328. DOI: 10.1053/crad.1999.0279

2. Vaiphei K, Singh P, Verma GR. Gallbladder malakoplakia in type 2 diabetes mellitus: a rare entity. BMJ case reports. 2012. Doi: 10.1136/ bcr-2012-006601

3. Dasgupta P, Womack C, Turner AG, Blackford HN. Malacoplakia: von Hansemann's disease. BJU international. 1999;84:464-169.

4. Wielenberg AJ, Demos TC, Rangachari B, Turk T. Malacoplakia presenting as a solitary renal mass. AJR Am J Roentgenol. 2004;183(6):1703-1705. DOI: 10.2214/ajr.183.6.01831703

5. van Crevel R, Curfs J, van der Ven AJ, Assmann K, Meis JF, van der Meer JW. Functional and morphological monocyte abnormalities in a patient with malakoplakia. Am J Med. 1998;105(1):74-77.

6. Di Tommaso L, Arizzi C, Roncalli M. Malacoplakia of the gallbladder. Histopathology. 2005;46(4):474-475. DOI: $10.1111 / \mathrm{j} .1365-$ 2559.2005.02012.x

7. Hanada M, Tujimura T, Kimura M. Cholecystic granulomas in gallstone disease. Acta Pathol Jpn. 1981;31(2):221-231.

8. Charpentier P, Prade M, Bognel C, Gadenne C, Duvillard P. Malacoplakia of the gallbladder. Human pathology. 1983;14(9), 827-828.

9. Singh K, Chatterjee T. A case of gallbladder mass: Malakoplakia (The tumor mimicker). Indian Journal of Pathology and Microbiology. 2017;60(1):122-124. Doi: 10.4103/0377-4929.200034

10.Webb M, Pincott JR, Marshall WC, Spitz L, Harvey BAM, Soothill JF. Hypogammaglobulinaemia and malakoplakia: response to bethanechol. European journal of pediatrics. 1986;145(4):297-302. 